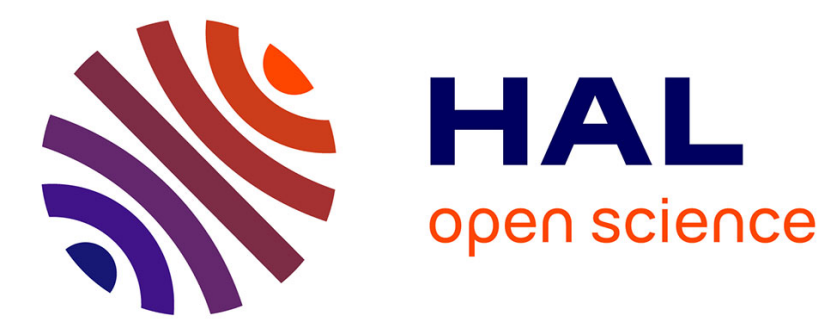

\title{
Analysis of grid file algorithms
}

Mireille Regnier

\section{To cite this version:}

Mireille Regnier. Analysis of grid file algorithms. RR-0369, INRIA. 1985. inria-00076187

\section{HAL Id: inria-00076187 \\ https://hal.inria.fr/inria-00076187}

Submitted on 24 May 2006

HAL is a multi-disciplinary open access archive for the deposit and dissemination of scientific research documents, whether they are published or not. The documents may come from teaching and research institutions in France or abroad, or from public or private research centers.
L'archive ouverte pluridisciplinaire HAL, est destinée au dépôt et à la diffusion de documents scientifiques de niveau recherche, publiés ou non, émanant des établissements d'enseignement et de recherche français ou étrangers, des laboratoires publics ou privés. 


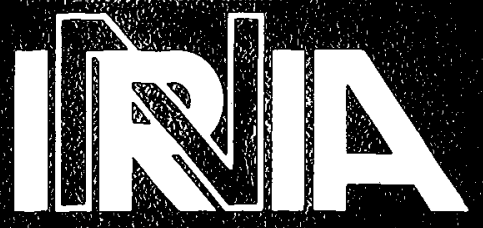

\section{Rapports de Recherche}

WCENTRE DE ROCOUENCOURT

$$
\text { No } 369
$$

\section{ANALYSIS OF GRID FILE ALGORITHMS}

Mireille REGNIER

Mars 1985 


\title{
ANALYSIS OF GRID FILE ALGORITHUS
}

\author{
Wireille REGNIER \\ INRIA \\ Rocquencourt \\ 78153-Le Chesnay (France)
}

Resume: Les algorithmes de grille, ou "grid-fle", ont et6 introduits dens [12] pour permettre un accis multiclef a des enregistrements dans un fichier croissant dynamiguement. Nous précisons ici deur algorithmes et calculons les expressions exactes des tailles moyennes des index correspondants. Nous effectuons ensuite une analyse asymptotique. Il apparait que la croissance des index pour des distributions uniformes n'est pas linbaire: $O\left(n^{1+\frac{1}{6}}\right)$ ou $O\left(n^{1+\frac{e-1}{b+1}}\right)$, ois s est le nombre d'attributs utilisés, $n$ le nombre de données, et - La capacite des pages. Finalement nous donnoins les résultats correspondants pour des distributions biaiskes et comparons les phases transitoires.

\begin{abstract}
Abutract: Orid fle algorithms were suggested in [12] to provide multi-key access to records in a dynamically growing file. We specify here two algorithms and derive the average sizes of the corresponding directories. We provide an asymptotic analysis. The growth of the indexes appear to be non linear for uniform distributions: $O\left(\nu^{1+\frac{1}{6}}\right)$ or $O\left(\nu^{1+\frac{s-1}{s b+1}}\right)$, where $s$ is the number of attributes being used, $\nu$ the file size, and b the page capacity of the system. Finally we give corresponding results for biased distributions and compare transient phases.
\end{abstract}

Keywords: dymamic data structures, multi-key acces, data bases, hashing, grid fle, analysis of algorithms, asymptotic analysis. 


\section{NNTRODUCTION}

We study here dymamic multidimensional structures for datas stored on secondary memory and accessed by an index. Several structures have been proposed as physical access methods in data bases systems $[13,1,12,8,16]$

In the first part, we specify two algorithms proposed in [12] under the generic name of grid flle. They generalize the standard single key retrieval search techniques and apply to the search of records characterized by several keys or attributes. The index is a multidimensional array which contains addresses of records on secondary memory. It may grow (and shrink) dynamically with the size of the set of records still ensuring a constant access cost to the fle:two disk accesses on the average. Several organizations are suggested in [12]. We specify and study two significant ones, that only depend on the values of the keys of the records and not on the order of insertion. They generalize Dynamic Hashing [9] and Extendible Hashing [3] to the multidimensional case and then we name them : Multidimensional Dynamic (resp. Extendible) Hashing and note MDH or MEH.

In the second part we derive exact expressions for the average values of the sizes of indexes for both organizations. In the third one, we derive asymptotic expansions. It appears that the growth is non linear. The proof is fairly involved and uses some elaborate asymptotic techniques (Mellin transform,Laplace method...). This problem was left unsolved in [12]. In the fourth part, we compare these methods for implementation of indexes. We study their asymptotic behaviours and their transient phases, when the data distribution is uniform or biased. As a conclusion, we discuss some implementation choices in relation to the capacity of pages in secondary memory.

\section{Grid File Algorithms}

We present in this section the so-called grid fle algorithms. We assume that secondary memory is divided in pages with a fixed capacity $b$ and that the system may allocate or remove these pages. Records are identified by $s$ attributes that form a key. We assume these attributes range in $[0,1]$. Thus, they are reals in $[0,1]$ or, equivalently, infinite sequences of bits $0-1$. Eventually, hashing the key would reduce to that case.

\subsection{Geometric partitions and grid file algorithms}

We describe the algorithms for two attributes, the generalization to $s>2$ is easily done. Records may be geometrically represented in the plane domain: $[0,1] \times[0,1]$, using the values of the attributes as coordinates. Every set of $n$ records is naturally associated to a partition of that space in regions that contain at most $b$ records. This partition is recursively defined by a call to PARTITION $([0,1],[0,1], 6,0,0)$ where the procedure PARTITION is described below. Two examples are given in Figure A.: 
(a)

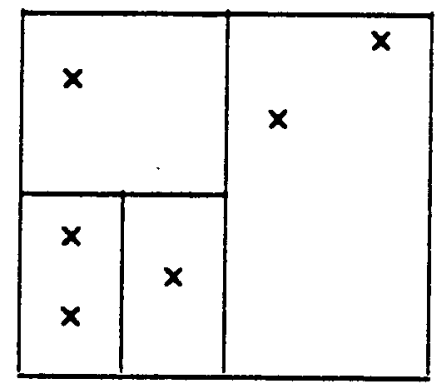

(b)

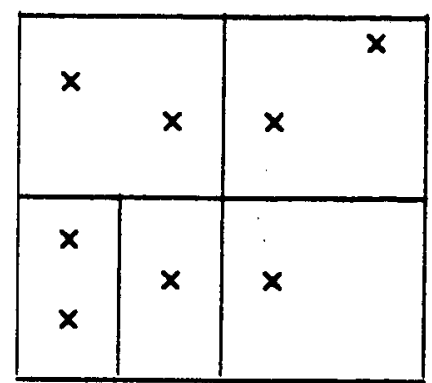

Fig.A.: Two possible partitions of $[0,1] \times[0,1]$, when $b=2$.

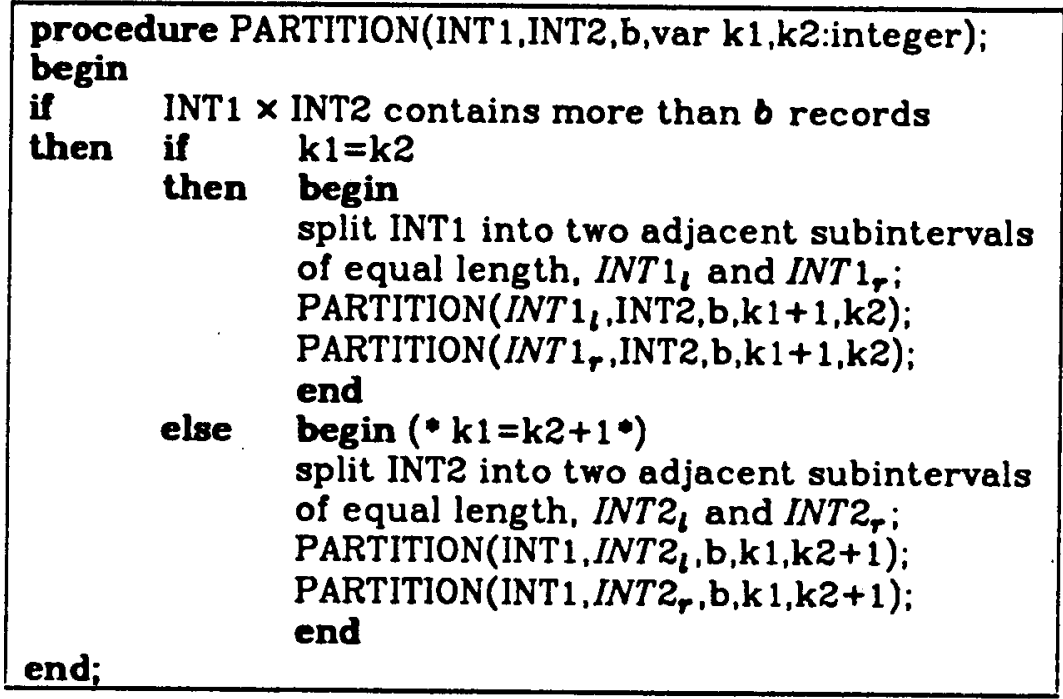

A region, INT $1 \times$ INT2 which contains more than $b$ records is split into two parts, according to the values of one attribute. A split where the first (resp. the second) attribute is involved may be called a vertical split, for it is represented in the space by a vertical (resp. horizontal) line. Notice that each final region has been created by splits using alternatively the first and the second attribute, beginning by the first one.

Such a partition describes the organization of secondary memory: a page is allocated for each element of the partition. Adresses of all the pages are kept in an index or directory that can be maintained dynamically. Two types of indexes are described in the following sections.

\subsection{Yultikey Extendible Hashing}

This construction of the directory is described in [16] as EXCELL method. Consider the element of the partition of smallest surface. "Embedding" the partition in a refined one where all the regions are of equal surface, one defines a directory as a 2-dimensional array containing pointers to secondary memory. The sets (a) and (b) in Figure A are associated to the indexes in Figure B. 
(a)

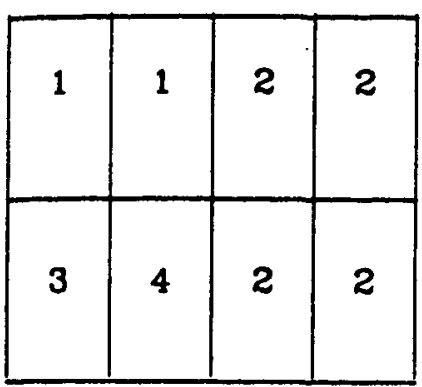

(b)

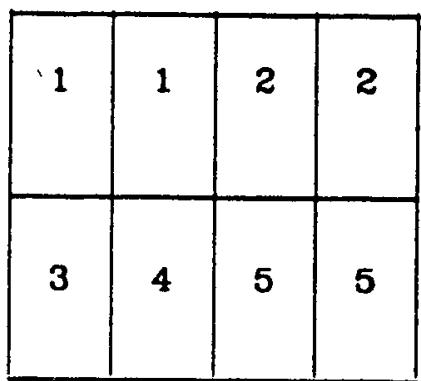

Fig.B.: $M E H$ indexes associated to Figure $A$.

Let us notice that some pages are addressed several times. This will make it easier to take into account some overflows and to allocate memory. To access some record, one uses the values of its attributes to determine an entry of the index. Then, following the pointer found in that entry, one retrieves the page containing the record sought. Thus, if the index may be kept resident in core, only one disk access is needed. As an other -and important-advantage, such a structure is suitable for dynamically varying sets of data with successive allocations of memory. These allocations are done step by step each time a new insertion causes overflow of an already allocated page. Such events are called collisions (see the terminology of classical hashing). At that time, one element of the partition contains more than b records and has to be split. The index is modified according to this refinement in two possible ways.

In the first -and easy- case, this refinement has no effect on the embedding as the new subdivision is already represented in the index. Thus, we only modify in it one (or possibly many) pointer to the overfow page $m 1$ in a pointer to a newly allocated page $m 2$. The records previously addressed to $m 1$ and the one which created the collision are shared between $m 1$ and $m 2$ according to the element of the new partition of the space they belong to. For instance, an insertion in the leftmost upper element in Figure A.b. creates a collision but does not modify the embedding. The next data set and index are represented in Figure $C$.

(a)

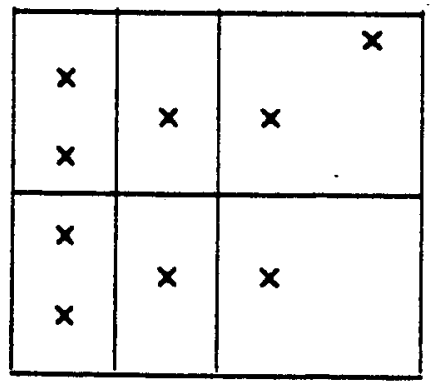

(b)

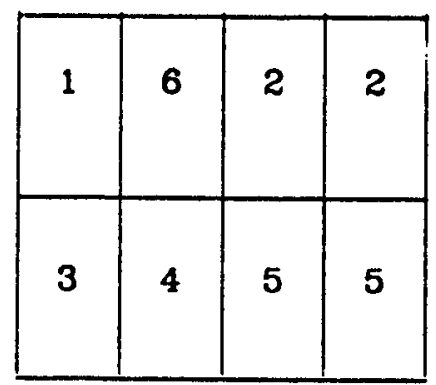

Fig.C.: After an insertion in Figure A.b.

Notice that we may have to modify several pointers, when an overflow page is addressed by more than two pointers. For instance, an insertion in the rightmost region in Figure A.a. would lead to a collision and the directory of Figure B.b.

In the second case, one has to modify the structure of the index. 
Whenever a collision occurs on an element of the partition of smallest size, one must double in size the directory, cop; $m$ pointers and finally update one of them. For instance, a collision on page 3 in Figure $C$ would lead to the new data set and index:

(a)

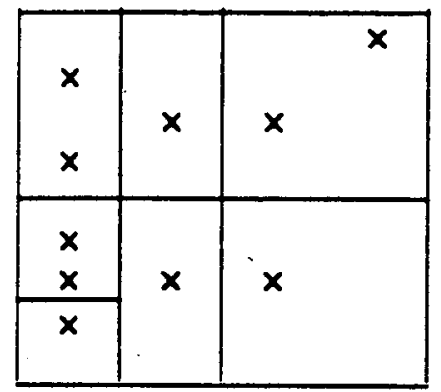

(b)

\begin{tabular}{|c|c|c|c|}
\hline 1 & 6 & 2 & 2 \\
\hline 1 & 6 & 2 & 2 \\
\hline 3 & 4 & 5 & 5 \\
\hline 7 & 4 & 5 & 5 \\
\hline
\end{tabular}

Fig.D.: Doubling in size the $M E H$ directory

One can see that for $s=1$, i.e. when records are characterized by a single key that range in $[0,1]$, this algorithm is Extendible Hashing (or EH) [3] .

\subsection{Yultikey Dynamic Hashing}

When the directory is constructed using MEH, it happens quite of ten that several pointers point to the same page. MDH tries to minimize the frequency of such events and save memory. Let us consider once more the partitions defined in 1.1. MDH index may be easily obtained from them. Roughly speaking, every line appearing in the partition is extended to the whole space. For instance. from Figure A., we get MDH indexes of Figure E.:

(a)

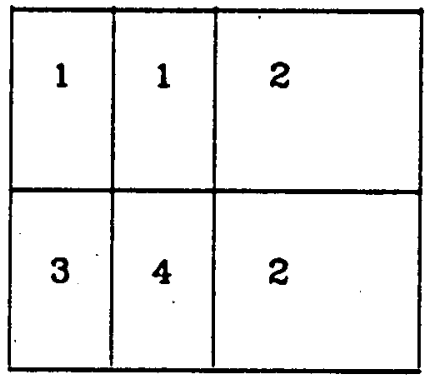

(b)

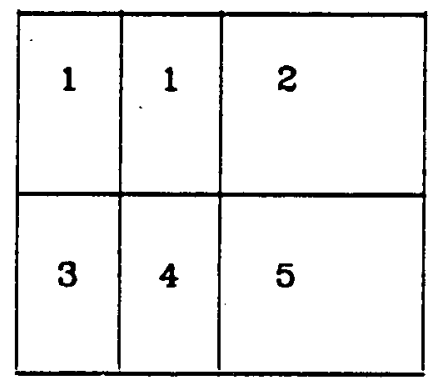

Fig.E.: $M D H$ indexes associated to Figure $A$.

As before, in retrieving a record one uses the values of its keys to choose an entry of the index. Again, one can distinguish between two types of collisions. In both cases, one new page is allocated and the (logical) partition is modified.

In the first case, the structure of the directory is not changed: informally, "no new line appears". One just modifies one (possibly several) pointers. For instance, an insertion in the rightmost part in A.a. would create the one of E.b. An insertion in A.b., creating the new data set in C.a. would create the MDH directory of Figure F.a. associated to the MEH directory in c.b. 
(a)

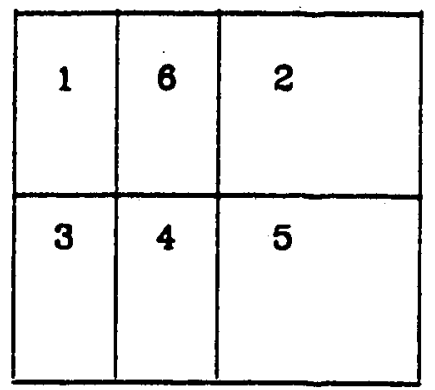

(b)

\begin{tabular}{|l|l|l|}
\hline 1 & 6 & 2 \\
\hline 3 & 4 & 5 \\
\hline 7 & 4 & 5 \\
\hline
\end{tabular}

Fig.F.: $M D H$ indexes associated to $M E H$ indexes of C.b. and D.b.

In the second case, collisions produce a modification of the structure and of the size of the index: "a new line appears".For example, if the data in C.a. is changed into the data set in D.a., a collision occurs on page 3 and we get the MDH directory in Figure F.b. associated to the MEH directory in D.b.

It is important to notice that this definition implies that a MDH directory is uniquely associated to a data distribution. It is a function of that distribution and it is independent of the history of insertions. We illustrate this assertion by an example. Assume a collision on page 5, after two more insertions. One should not use the preexisting horizontal line creating two entries to page 5 (see in Figure G.a. such an impossible directory). But one forces a vertical line to be drawn and get the directory in Figure G.b. Then, one may verify that this index is the one that would have been obtained if the collision on page 5 had occurred before the collision on page 3 .

(a)

\begin{tabular}{|l|l|l|}
\hline 1 & 6 & 2 \\
\hline 3 & 4 & 5 \\
\hline 7 & 4 & 8 \\
\hline
\end{tabular}

(b)

\begin{tabular}{|c|c|c|c|}
\hline 1 & 6 & 2 & 2 \\
\hline 3 & 4 & 5 & 8 \\
\hline 7 & 4 & 5 & 8 \\
\hline
\end{tabular}

Fig.G.: Independency of the history of insertions.

Notice that MDH is a generalization of Dynamic Hashing (we note DH) [9] .

To sum up, we may say that both MDH and MEH directories are associated to geometric partitions of the space and are independent of the history of insertions. They can manage dynamically growing sets. For each collision, a page in secondary memory is allocated, and the directory is modified according to the type of collision. In the next sections, we are dealing with performances. It is pointed out in [16] that the directory of $\mathrm{MEH}$ is the directory of Extendible Hashing on one key obtained by shuffling the $s$ keys. The performances of EH (and, consequently, of $\mathrm{MEH}$ ) have been studied in $[5,10,14,17]$. They are resumed in Theorem A:

Theorem A: When the data are uniformly distributed according to a Bernoulli or Poisson law, the average size of the directory of $E H$ or $M E H$ satisfies asymptotically: 


$$
S_{U E H}(n)=P\left(\left(\left(1+\frac{1}{b}\right) \log _{2}(n)\right\}\right) n^{1+\frac{1}{b}}+O(n) .
$$

where $P$ is a continuous periodic function with period 1 and mean:

$$
\frac{1}{\log 2}[(b+1) !]^{\frac{-1}{b}} \Gamma\left(1-\frac{1}{b}\right) \text {. }
$$

Moreover, the average load factor of the data part a fuctuates around log2, or $p \log \frac{1}{p}+q \log \frac{1}{q}$ when the distribution is biased.

We study MDH below.As the occupation of the file on secondary memory is obviously the same for MDH and MEH, we are dealing only with the size of the $\mathrm{MDH}$ directory. Moreover, when $\mathrm{MDH}$ is used, some information about splits on the attributes should be kept in core. One creates axial directories (see a possible implementation in [11]). Their sizes are studied below. In the third part, we will give some results about directories sizes in the biased case for both algorithms.

\section{Analysis of grid file}

\subsection{The Statistical Yodel.Poisson Approximation}

We have not specified yet the type of the keys (sequences of characters or bits,integers,...) nor their repartition on the set of possible values. In this analysis, we assume that we have $s$ hashing functions $h_{i}$. Each $h_{i}$ associates to any key of the attribute $i$ a sequence of bits, called pseudokey. In the following we assimilate the key to the pseudokey. Thus, a value on one attribute is represented by an infinite sequence of bits which itself can be considered as a real number over the interval $[0,1]$. We assume now that in all the successive partitions of $[0,1]$ any subinterval is divided in two subintervals of equal lengths (see the buddy system in [3] ), or remains inchanged. Then we must use one more bit to address the keys in a split subinterval. Moreover, we assume uniformity of pseudokeys. This is made precise in the following.

We generalize in the multidimensional case some hypotheses on the distribution of the keys, that are classical when we hash with one key. In the problems studied, the keys are distributed on a finite product of intervals and the analysis can be done when we know the distribution of the keys hashed to $r$ disjoint volumes. In hashing, this distribution on intervals follows a Bernoulli law, or a multinomial law or a Poisson law, whose parameters are functions of the lengths of the intervals considered. In multidimensional hashing, we show how the distribution law on $r$ disjoint volumes results in a simple way from the distributions laws on the attributes. The results are quite analogous to the monodimensional ones, the parameters length of intervals are now the volumes (or the surfaces when $s=2$ ).

Let $n$ be the number of records in the file. We make two hypotheses on the distribution on the attributes of the keys or pseudokeys.

(i) the values of the keys on the $s$ attributes are independent.

(ii) for any attribute $i$, for any $j$, the bit $b_{j}^{j}$ satisfies either:

$$
\text { (a) } \operatorname{Pr}\left(b_{j}^{i}=0\right)=\operatorname{Pr}\left(b_{j}^{i}=1\right)=\frac{1}{2}
$$


(b) $\operatorname{Pr}\left(b_{j}=0\right)=p$. $\operatorname{Pr}(b j=1)=q, p+q=1$

In (a) the distribution is uniform, in (b), it is biased.

The records are distributed on volumes. We define a distribution law on elementary volumes (in the borelian meaning) which are products of segments $I_{1} \times \cdots \times I_{s}$ with dimensions $p_{1}, \ldots, p_{s}$. Then:

\section{Proposition 1:}

With the hypotheses ( $i$ ) and (ii-a), we have:

(a) The random variable $J$ of the number of keys hashed to an elementary volume $I$ of dimensions $p_{1}, \ldots, p_{\text {s }}$ follows a Bernoulli law with parameters $\left(n, p_{1} \times \cdots \times p_{s}\right)$.

(b) If $I_{1}, \ldots, I_{r}$ are disjoint volumes with volumes $v_{1}, \ldots, v_{r}$, and $J_{1}, \ldots, J_{r}$ are the corresponding r.v.:

$$
P\left(J_{1}=j_{1} \ldots, J_{r}=j_{r}\right)=\left[j_{1}, \ldots, j_{r}, n-j \mid v_{1}^{j_{1}} \ldots v_{r}^{j_{r}}(1-v)^{n-j}\right.
$$

with $j=j_{1}+\ldots+j_{r}, v=v_{1}+\ldots+v_{r}$.

The first assumption is readily proved and the associated generating function is, when $s=2$ :

$$
\varphi(t)=\sum_{j=0}^{\infty} P(J=j) t^{j}=\left[p_{1} p_{2} t+\left(1-p_{1} p_{2}\right)\right]^{n} .
$$

The assertion (b) may be proved using the properties of compound distributions[4,15,ch.Il ] The generating function associated to $r$ disjoint volumes is:

$$
\psi\left(t_{1}, \ldots, t_{r}\right)=\left[\sum_{i=1}^{r} v_{i} t_{i}+(1-v)\right]^{n}
$$

and (b) follows.

To simplify the calculations, we make a Poisson approximation in multidimensional case.

Proposition 2: Let us assume that the number of records in the file follows a Poisson law with mean $\nu$. Then, the random variables $J_{1}, \ldots, J_{r}$ of the number of records hashed to $r$ disjoint blocks $I_{1}, \ldots, I_{r}$ of volumes $v_{1}, \ldots, v_{r}$ are $r$ independent random variables, following Poisson laws with parameters. $\nu v_{1}, \ldots, v_{r}$.

This may be proved using again the properties of compound laws. In the following. we assume the Poisson hypotheses on the number $n$ of records and we use the probabilistic laws defined in Proposition 2.

Remark 1: Biased distributions define a new measure on $[0,1]^{5}$. Proposition 2 still holds if we generalize the notion of volume. Consider only surfaces $2^{\text {ud }} \times 2^{\text {hd }}$ obtained by splits of intervals into 2 parts. All records adressed to such a surface $S$ have keys of the form: $\left(b_{1} \ldots b_{\text {vd }} \ldots, c_{1} \ldots c_{\text {hd }} \ldots\right)$ with identical sequences $\left(b_{i}\right)$ and $\left(c_{i}\right)$. In the uniform case, the distribution on $S$ only depends on the sum vd thd (see above), in the biased case one uses two more parameters $k_{1}$ and $k_{2}$, the number of 0 -bits in sequences $\left(b_{i}\right)$ and $\left(c_{i}\right)$. These parameters also count the number of left vertical (resp. horizontal) splits performed to "draw this rectangular surface". According to hypotheses (ii.b), the number of records addressed to $S$ is $\nu p^{k_{1}} q^{\nu d-k_{1}} p^{k_{2}} q^{h d-k_{z}}$ and one may call "volume" the quantity: $p^{k_{1}} q^{\text {vd }-k_{1}} p^{k_{z}} q^{\text {nd }-k_{z}}$. 
Remark 2: This approximation is justified by the fact that Poisson and Bernoulli results are asymptotically equivalent.

\subsection{Average Sizes of Axial Directories:}

In this section, we derive the exact expressions of the average values of axial directories, under the probabilistic Poisson hypotheses defined in section 2.1 .

Let us give some notations. A subdivision of the interval of values of one attribute may be graphically represented by a straight line, vertical or horizontal, depending on the attribute. Then we shall talk of lines rather than subdivisions. We have already defined the depth of a subdivision or a line. Notice that a line at depth $k$ divides any row or column of width $2^{-k}$ in two rows or columns of width $2^{-(k+1)}$. This column (or row) contains records from the data set with keys of the form: $b_{1} \ldots b_{k}$. Let the left aplit number be the number of 0 -bits in this sequence. For example, the dotted vertical or horizontal line in Figure 2.2. are associated to 1 and 0 while their depths are 1 and 2 .

In all the paper, $\log x$ stands for $\log _{2} x$.

\section{Theorem 1}

The expectation of the number of vertical subdivisions is in the uniform case:

$$
E_{V}(\nu)=\sum_{\nu d \geq 0} 2^{v d}\left(1-\rho_{0}\left(\nu 2^{-2 v d}\right)^{2^{v e}}\right) \text {. }
$$

or, in the biased case:

$$
E_{V}(\nu)=\sum_{k \geq 0 k} \sum_{1=0}^{k}\left(\begin{array}{c}
k \\
k 1
\end{array}\right)\left(1-\prod_{k 2} f_{0}\left(\nu p^{k 1+k 2} q^{k-k 1+k-k 2}\right)^{\left(\begin{array}{l}
k \\
k 2
\end{array}\right)}\right)
$$

The expectation of the number of horizontal subdivisions is:

$$
E_{H}(\nu)=\sum_{n d z 0} 2^{h d}\left(1-f_{b}\left(\nu 2^{-2 h d-1}\right)^{2^{N+2}}\right) .
$$

or, in the biased case:

$$
E_{H}(\nu)=\sum_{k \geq 0 k} \sum_{1=0}^{k}\left(\begin{array}{c}
k \\
k 1
\end{array}\right)\left(1-\prod_{k=2} f_{0}\left(\nu p^{k 1+k 2} q^{k-k 1+k+1-k 2}\right)^{\left(\begin{array}{c}
k+1 \\
k 2
\end{array}\right)}\right)
$$

The expectation of entries in the vertical (resp. horizontal) index is:

$$
N_{V}(\nu)=1+E_{V}(\nu) \text {. }
$$

fesp. $\left.N_{H}(\nu)=1+E_{H}(\nu)\right)$.

To prove the theorem, we estimate, for all the possible subdivisions, their probabilitie to exist.We get:

\section{Lemma 1:}

Let $\Delta_{V}$ (resp. $\Delta_{H}$ ) be a vertical (resp. horizontal) line at depth $k$, with left split number $k_{1}$. We have, in the uniform case:

$$
\begin{gathered}
P\left(\Delta_{V}\right)=1-\rho_{0}\left(\nu 2^{-2 \nu d)^{2 \omega}}\right. \\
P\left(\Delta_{H}\right)=1-\rho_{0}\left(\nu 2^{-2 n d-1}\right)^{2^{N+1}} .
\end{gathered}
$$

and, in the biased case: 


$$
\begin{gathered}
P\left(\Delta_{V}\right)=\left(1-\prod_{k_{2}} f_{0}\left(\nu p^{k_{1}+k_{2}} q^{k-k_{1}+k-k_{2}}\right)^{\left(\begin{array}{l}
k \\
k_{1}
\end{array}\right)}\right. \\
P\left(\Delta_{H}\right)=\left(1-\prod_{k_{2}} f_{0}\left(\nu p^{k_{2}+k_{2}} q^{k-k_{1}+k+1-k_{2}}\right)^{\left(\begin{array}{l}
k_{1}+1 \\
k_{2}
\end{array}\right)}\right.
\end{gathered}
$$

Proof: We establish Lemma 1 for the vertical subdivisions. We first consider the uniform case.

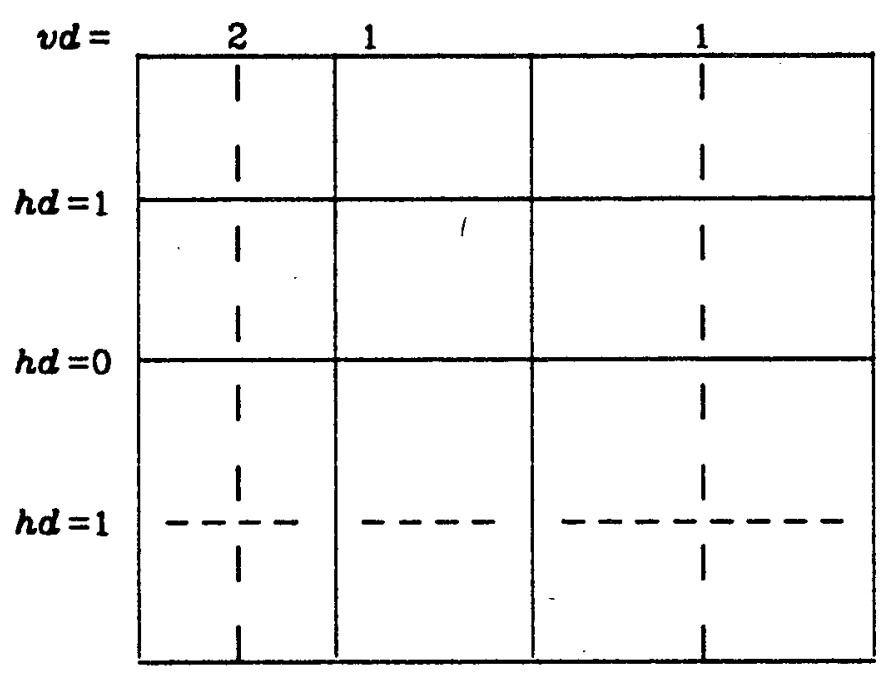

Figure 2.2.a

A vertical line $\Delta$ at depth $v d$ would split some associated column of width $2^{-2 d}$. Such a column may be logically divided in $2^{\text {vd }}$ squares of dimensions $2^{-v d} \times 2^{-v d}$. (see for example the dotted vertical line when $v d=2$ ). Considering the partition law, one may easily be convinced that such a line is required if at least one of these squares contains more than $b$ elements. Now the probability that such a square of surface $2^{-2 v d}$ contains at most $b$ elements is, according to Proposition 2:

$$
\sum_{i=0}^{b} e^{\nu 2^{-v v}} \frac{\left(\nu 2^{-2 \nu d}\right)^{i}}{i !}
$$

and we consider $2^{\text {vd }}$ disjoint square surfaces. As the distributions are independent, the probability that no surface contains more than $b$ elements is: $f_{0}\left(\nu 2^{-2 v d)^{2 \omega t}}\right.$ and we get:

$$
E\left(\Delta_{V}\right)=1-\rho_{0}\left(\nu 2^{-2 \nu d)^{2 \nu d}} .\right.
$$

We derive in the same manner the results on horizontal lines, considering $2^{\text {hd }+1}$ rectangles of dimensions $2^{-h d} \times 2^{-(h d+1)}$ (see for example the dotted line for $h d=1$ ).

Theorem 1 follows immediately. There are $2^{\text {vd }}$ possible lines at depth $v d$ and the expectation of the number of subdivisions is obtained by summation.

We are dealing now with biased distributions. Consider again the $2^{k}$ squares associated to a vertical line $\Delta_{V}$ at depth $k$. For all records in a given 
square, the second attribute keys start with the same sequence: $b_{1} \ldots b_{k}$, with $k_{2}$ 0-bits. Then the volume is: $p^{k 1} q^{k-k 1} p^{k 2} q^{k-k 2}, k 2=0 . . k$, and the probability that it contains less than b records is: $\rho_{0}\left(\nu p^{k i+k 2} q^{k-k i+k-k 2}\right)$. As distributions are independent, we get:

$$
P\left(\Delta_{V}\right)=\prod_{k 2=0}^{k} f_{0}\left(\nu p^{k 1+k 2} q^{k-k 1+k-k 2}\right)\left(\begin{array}{c}
k+1 \\
k_{1}
\end{array}\right)
$$

Theorem 1 follows. The expectation of the number of subdivisions is obtained by summation. There are $2^{k}$ possible lines at depth $k_{\text {, and }}\left(\begin{array}{l}k \\ k_{1}\end{array}\right)$ among them with a parameter $k 1$.

\section{3..Average Sizes of Main Indexes:}

We derive now the expression of the average size of the index. The main result is given in Theorem 2 .

Theorem 2:

The total number of grid blocks is, on the average:

$$
S_{U D H}(\nu)=N_{H}(\nu) \cdot N_{V}(\nu)-T(\nu)-1
$$

where $N_{H}$ and $N_{V}$ are the average sizes of axial directories, and $T(\nu)$ is, in the uniform case:

$$
\begin{aligned}
& \sum_{\nu d, h d \geq 0} 2^{\nu d+h d} f_{0}\left(\nu 2^{-2 \nu d}\right)^{2^{\nu d}} \rho_{0}\left(\nu 2^{-2 h d-1}\right)^{2^{h d+1}}
\end{aligned}
$$

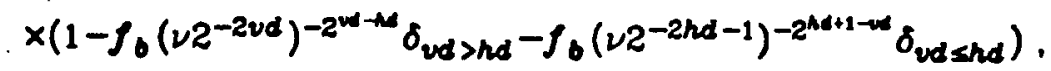

and, in the biased case:

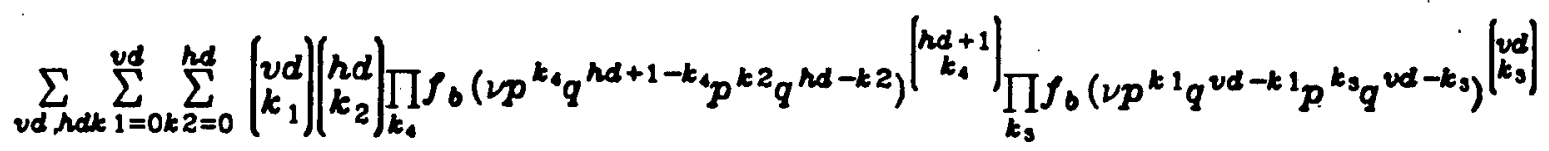

$$
\begin{aligned}
& \left(1-\frac{\delta_{v d>h d}}{\prod_{m=0}^{u d-h d} f_{b}\left(\nu p^{k 1} q^{v d-k 1_{p} k 2+m} q^{\nu d-k 2-m}\right)^{(v d-h d)}}\right. \\
& \left.-\frac{\delta_{u d \leq h d}}{\prod_{m=0}^{n d+1-v d} f_{0}\left(\nu p^{k 1+m} q^{h d+1-m} p^{k 2} q^{h d-k 2}\right)^{(h d+1-v d)}}\right)
\end{aligned}
$$

To prove this, we first remark that the number of grid blocks is the number of intersections of lines. Thus we study in Lemma 2 the probability that the intersection of a vertical line $\Delta_{V}$ at depth $v d$ with parameter $k 1$ and of a horizontal line $\Delta_{H}$ at depth $h d$ with parameter $k 2$ exist.

\section{Lemma 2:}

Let $\Delta_{V}$ and $\Delta_{H}$ be two vertical and horizontal lines (or subdivisions). Let $v d$ and hd be their depths, $k 1$ and $k 2$ their left split numbers. We note 
$P\left(\left(n \circ \Delta_{V}\right) \cap\left(n \circ \Delta_{H}\right)\right)$ the probability that none of them exist. This quantity is, for uniform or biesed distributions :

(i) if $v d>h d$ :

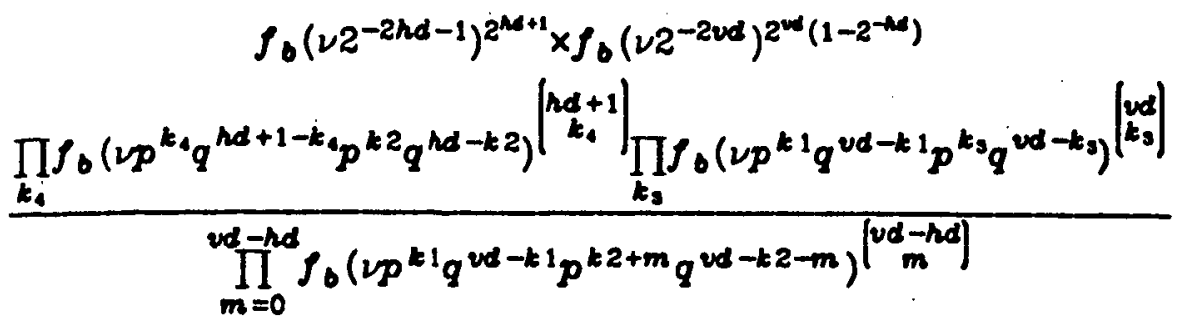

(ii) if $v d \leq h d$ :

$$
\begin{aligned}
& f_{0}\left(\nu 2^{-2 \nu d}\right)^{2 \omega} \times \rho_{0}\left(\nu 2^{-2 h d-1}\right)^{2^{N+1}\left(1-2^{-\infty}\right)}
\end{aligned}
$$

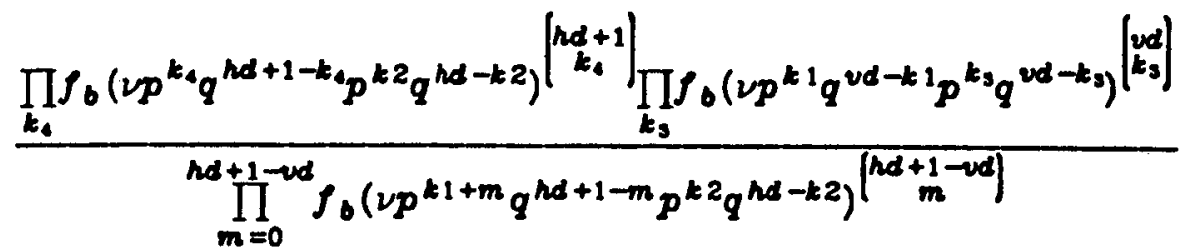

Proof of Lemma 2: We derive the result when $v d s h d$ : see the intersection of dotted lines in Figure 2.2. We write:

$$
P\left(\left(n \circ \Delta_{V}\right) \cap\left(n \circ \Delta_{H}\right)\right)=P\left(n \circ \Delta_{V}\right) \cdot P\left(\left(n \circ \Delta_{H}\right) /\left(n \circ \Delta_{V}\right)\right) \text {. }
$$

where $P\left(\left(n \circ \Delta_{V}\right) \cap\left(n \circ \Delta_{H}\right)\right)$ is the probability that neither $\Delta_{V}$ nor $\Delta_{H}$ exist. The line $\Delta_{V}$ (resp. $\Delta_{H}$ )does not exist iff none of the rectangles in the surfaces (1) and(2) contains more than $b$ elements. As surface (1) is included in a square defined by $\Delta_{V}$-and thus unsplit- we get:

$$
\begin{aligned}
P\left(\left(\text { no } \Delta_{H}\right) /\left(\text { no } \Delta_{V}\right)\right) & =P(\text { no rectangle in }(2) \text { contains more than b elements }) \\
& =\frac{P\left(\Delta_{H}\right)}{P(\text { no rectangle in (1) has more than b elements })}
\end{aligned}
$$

When the distribution is uniform, all the rectangles have the same volume: $2^{-2 h d-1}$ and there are $2^{\text {hd }+1-v d}$ of them in (1). The result is then established. When it is biased, the volumes are different. For all records in rectangles defined by $\Delta_{H}$. the second attribute keys have the hd first bits identical, with $k 20$-bits. For rectangles in (1). the first attribute keys have the $v d$ first bits identical. As they are included in the surface defined by $\Delta_{Y}$ they have $k 10$ bits. Moreover, the next $h d+1-v d$ that also characterize them contain $m 0$ bits. Thus the volumes are: $p^{k 1+m} q^{h d+1-k 1-m} p^{k 2} q^{v d-k 2}$, and Lemma 2 follows.

Proof of Theorem 2: We note $P\left(\Delta_{V} \cap \Delta_{H}\right)$ the probability that $\Delta_{V}$ and $\Delta_{H}$ both exist. As:

$$
P\left(\Delta_{V} \cap \Delta_{H}\right)=P\left(\Delta_{V}\right)+P\left(\Delta_{H}\right)+P\left(\left(n \circ \Delta_{V}\right) \cap\left(n \circ \Delta_{H}\right)\right)-1 .
$$

summing over all the possible intersections, we get Theorem 2 . For example, for a uniform distribution and $v d \leq h d$, we write:

$$
\begin{aligned}
P\left(\Delta_{V} \cap \Delta_{H}\right)= & \left(1-f_{0}\left(\nu 2^{-2 \nu d}\right)^{2^{\nu \alpha}}\right) \times\left(1-f_{0}\left(\nu 2^{-2 n d-1}\right)^{2^{N+1}}\right) \\
& -f_{0}\left(\nu 2^{-2 n d-1}\right)^{2^{N+1}} f_{b}\left(\nu 2^{-2 \nu d}\right)^{2^{\nu \alpha}}\left(1-f_{0}\left(\nu 2^{-2 h d-1}\right)^{-2^{N+1-\alpha}}\right) .
\end{aligned}
$$


There are $2^{u d+h d}$ possible pairs of lines at depths $v d$ and $h d$.Thus, we count $2^{v d+h d}$ intersections of lines $\left(\Delta_{V}, v d\right)$ and $\left(\Delta_{H}, h d\right)$. We count too half of the intersections with the edges. Finally, considering also the case $v d>h d$, we get:

$$
\begin{aligned}
S_{\nu D H}(\nu)= & \sum_{\nu d, h d \geq 0} 2^{\text {vd }+h d}\left(1-\rho_{b}\left(\nu 2^{-2 v d}\right)^{2^{\nu d}}\right)\left(1-f_{b}\left(\nu 2^{-2 n d-1}\right)^{2^{\Lambda d}}\right)+E_{H}(\nu)+E_{V}(\nu)-T(\nu) \\
& =\left(E_{H}(\nu)+1\right) \times\left(E_{V}(\nu)+1\right)-T(\nu)-1=N_{H}(\nu) \times N_{V}(\nu)-T(\nu)-1
\end{aligned}
$$

\section{Asymptotic behaviour}

The expressions we derived in Section 2 are quite intricated. In order to know the variations of these quantities with the number $n$ of records, we study their asymptotic expansions. The techniques involve both Mellin transform methods and the Laplace method for integrals. We recall some general principles in Section 3.1. More details are given in[6]. Then we study in 3.2. axial directories sizes, $N_{V}(\nu)$ and $N_{H}(\nu)$. Finally, in 3.3. we prove that the main directory size $S_{U D H}(\nu)$ is asymptotically equivalent to $N_{V}(\nu) . N_{H}(\nu)$.

\subsection{Mellin Transform:}

The Mellin transform of a continuous function $f:[0,+\infty[\rightarrow R$ is:

$$
f^{*}(s)=\int_{0}^{\infty} f(x) x^{s-1} d x=\mathbf{M}(f, s)
$$

We have:

$$
\left\{\begin{array}{l}
(f+g)^{*}(s)=f^{\bullet}(s)+g^{\bullet}(s) \quad(i) \\
\mathbf{U}(f(\alpha x), s)=a^{-s} \mathbf{U}(f, s)
\end{array}\right.
$$

The asymptotic expansion of such a function $f$ is related to the singularities of its Mellin transform $f^{*}$. A pole $\alpha$ of $f^{*}$ of order $k$, with the expansion, as $s \rightarrow-\alpha .:$

$$
f^{\prime}(s)=\frac{a_{0}}{s+a}+\cdots+\frac{a_{k-1}}{(s+a)^{k}}
$$

is associated [2] in the asymptotic development of $f$ to the term:

$$
\left(a_{0}+\ldots+a_{k-1}(\log x)^{k-1}\right) x^{-\alpha} \text {. }
$$

In the following, we are dealing with harmonic power sums, i.e. functions $F$ of the form:

$$
F(x)=\sum_{k} \alpha_{k} f_{b}\left(\beta_{k} x\right)^{\gamma_{k}} \times g\left(\beta_{k} x\right)=\sum_{k} \alpha_{k} h_{\gamma_{k}}\left(\beta_{k} x\right)
$$

where: $\boldsymbol{\gamma}_{k} \rightarrow \infty$.

If we proceed formally, we get:

$$
F^{*}(s)=\sum_{k} \alpha_{k} \beta_{k}^{-s} h_{\gamma_{k}} \cdot(s) .
$$

To simplify the study of the series and derive the analytic properties of $F^{\bullet}$, we find an asymptotic expansion of the integral: 


$$
h_{t}^{*}(s)=\int_{0}^{\infty} e^{-t \log f_{0}(x)} g(x) x^{s-1} d x
$$

as a function of $t$. Under some conditions of regularity for $f$ and $g$, such an expansion can be given and depends only on the expansion of $f$ and $g$ around 0 . We have here:

$$
\left\{\begin{array}{c}
\log f(x) \sim-c x^{\alpha}+\alpha x^{\beta}, a \neq 0 \\
g(x) \sim 1+f x^{\gamma},
\end{array}\right.
$$

we get, using the Laplace method or the Watson-Doetsch lemma [7] :

$$
h_{t} \cdot(s) \sim g_{t}(s)
$$

with:

$$
g_{t}(s)=\frac{1}{\alpha} \Gamma\left(\frac{s}{\alpha}\right)(c t)^{-\frac{s}{\alpha}}
$$

Then, accordingly to the relation above between (i) and (ii), we derive the asymptotic properties of $F^{\circ}$ from those of

$$
G^{*}(s)=\sum_{k} \alpha_{k} \beta_{k}^{-s} g_{\eta_{k}}(s) \text {. }
$$

\subsection{Asymplotic Behaviour of Axial Directories Sizes:}

The $s$ attributes are numbered $1, \ldots, j, \ldots, s$ according to their order of splitting. Thus:

Theorem 3: The expected sizes of axial directories satisfy asymptotically:

where:

$$
\begin{gathered}
N_{V}(\nu) \sim \nu^{\frac{1}{2}\left(1+\frac{1}{2 b+1}\right)} A_{b} P\left(\left\{\frac{1}{2}\left(1+\frac{1}{2 b+1}\right) \log \nu\right\}\right)+O\left(\nu^{\frac{1}{2}\left(1-\frac{1}{2 b+1}\right)}\right), \\
N_{H}(\nu) \sim 2^{-\frac{b}{2 b+1} \nu^{\frac{1}{2}\left(1+\frac{1}{2 b+1}\right)}} A_{b} P\left(\left\{\frac{1}{2}\left(1+\frac{1}{2 b+1}\right) \log \nu\right\}\right)+O\left(\nu^{\frac{1}{2}\left(1-\frac{1}{2 b+1}\right)}\right) .
\end{gathered}
$$

$$
\mu=\frac{1}{2 b+1 .} A_{b}=\frac{1}{\ln 2}[(b+1) !]^{-\mu} \Gamma(1-\mu) .
$$

$P(u)$ is a fuctuating function with small amplitude and period 1:

with:

$$
P(u)=\sum_{l=\infty}^{\infty} c_{l} e^{-2 u m u}
$$

Proof:

$$
c_{l}=[(b+1) !]^{x_{l}} \frac{\Gamma\left(-\mu+\chi_{l}\right) \mu}{[\ln 2 \Gamma(1-\mu)]}, x_{l}=\frac{2 i l \pi \mu}{\ln 2}
$$

We prove the theorem for $N_{V}(\nu)$. We have seen in section 2.2. that the expected size of the first axial directory is:

$$
N_{V}(\nu)=1+\sum_{\nu d \geq 0} 2^{v d}\left(1-f_{0}\left(\nu 2^{-2 v d}\right)^{2^{\nu d}}\right)
$$

To get its asymptotic expansion, we integrate the asymptotic expansion of:

$$
\frac{d}{d \nu} N_{V}(\nu)=\frac{\nu^{b}}{b !} \sum_{\nu d} 2^{-2 b(\nu d)} f_{b}\left(\nu 2^{-2 \nu d}\right)^{2^{\nu}} \times \frac{1}{e_{b}\left(\nu 2^{-2 \nu d}\right)} .
$$


We define $F(\nu)$ by :

$$
\frac{d}{d \nu} N_{V}(\nu)=\frac{\nu^{b}}{b !} F(\nu)
$$

and we study its Mellin transform.

$F(\nu)$ may be written:

$$
F(\nu)=\sum_{k} \alpha_{k} f_{0}\left(\beta_{k} \nu\right)^{\gamma_{t}} \quad x g\left(\beta_{k} \nu\right)=\sum_{k} \alpha_{k} h_{\gamma_{1}}\left(\beta_{k} \nu\right)
$$

We have here:

We write:

$$
\left\{\begin{array}{c}
\log f_{b}(x)=\log \left[e_{b}(x) e^{-x}\right] \sim-\frac{1}{(b+1) !} x^{b+1}+\frac{b+1}{(b+2) !} x^{b+2} \\
g(x)=\frac{1}{e_{b}(x)} \sim 1-x .
\end{array}\right.
$$

We note:

$$
G^{\bullet}(s)=\frac{1}{b+1} \Gamma\left(\frac{s}{b+1}\right)[(b+1) !]^{\frac{s}{b+1}} \sum_{k} 2^{k \frac{a(2 b+1)-2 b(b+1)}{b+1}}
$$

$$
\mu=\frac{1}{2 b+1} \cdot \zeta=1+\frac{1}{2 b+1} .
$$

The series $G^{\bullet}$ is analytic in the stripe

$$
0<\operatorname{Re}(s)<s_{1}=\frac{2 b(b+1)}{2 b+1}=b \times \zeta
$$

Moreover, $G$ is meromorphic in $\langle 0,+\infty\rangle$. Then, one can show [6] that $F^{*}$ is defined in $\left\langle 0, s_{1}\right\rangle$, is analytic there, and meromorphic in some larger stripe $\left\langle 0, s_{2}\right\rangle$. Its poles in the stripe $\left\langle 0, s_{2}\right\rangle$ are:

$$
p_{l}=6 \zeta+\frac{i l \pi \zeta}{\ln 2}, l \in \mathrm{Z}
$$

Notice that they are regularly distributed on the vertical axis: $\operatorname{Re}(s)=s_{1}$. The residues at these poles are:

$$
r_{l}=\frac{\mu}{\ln 2} \Gamma\left(1-\mu+\chi_{l}\right)[(b+1) !]^{20 \mu} .
$$

with:

$$
x_{l}=\frac{2 i l \pi \mu}{\ln 2} \text {. }
$$

Then, using (i) and (ii) above, we get: $F(\nu)=\sum_{l=-\infty}^{+\infty} r_{l} \nu p_{l}$

and: $\frac{d}{d \nu} N_{V}(\nu)=\frac{\nu^{b}}{b !} \sum_{l=-\infty}^{+\infty} r_{l} \nu^{-p_{l}}$.

Integrating this expression, we get the result. The order of approximation is a function of the next terms of the developments of $f_{0}$ and $g$ in 0 , which define the value $s_{2}$ above $[6,15]$.

We have a similar result when we use $s$ attributes. The $s$ attributes are numbered $1, \ldots, j, \ldots, s$ according to their order of splitting. Thus:

Theorem 3Bis: The expected size of an axial directory associated to the attribute $j$ satisfies, asymptotically: 


$$
N_{j}(\nu) \sim 2^{-\diamond(j-1) \eta_{\nu} \frac{1+(s-1) \eta}{s}} A_{b} P\left(\left\{\frac{1+(s-1) \eta}{s} \log \nu\right\}\right)+O\left(\nu^{\frac{1-(s-1) \eta}{s}}\right) .
$$

where:

$$
\eta=\frac{1}{s b+1 .} A_{b}=\frac{1}{\ln 2}[(b+1) !]-\eta \Gamma(1-\eta) .
$$

$P(u)$ is a fluctuating function with small amplitude and period 1:

$$
P(u)=\sum_{l=\infty}^{\infty} c_{l} e^{-2 u m u} \text {. }
$$

with:

$$
c_{l}=[(b+1) !]^{x_{l}} \frac{\Gamma\left(-\eta+\chi_{l}\right) \eta}{[\ln 2 \Gamma(1-\eta)]}, x_{l}=\frac{2 i l \pi \eta}{\ln 2}
$$

\subsection{Main Indexes:}

In section 2, we derived $S_{U D H}(\nu)=N_{V}(\nu) \cdot N_{H}(\nu)+T(\nu)$. We show here that $T$ is negligeable when compared to $N_{V} . N_{H}$. The meaning of this result is that the average size of the index is the product of the average sizes of the axial directories. This result still holds for $s>2$. We establish it first for uniform distributions.

Theorem 4:

The average size of the index of $M D H$ satisfies, for a unifurm distribution

$S_{U D H}(\nu)=\nu^{1+\frac{s-1}{s b+1}} c_{b} Q\left(\left\{\frac{1}{s}\left(1+\frac{1}{s b+1}\right) \log _{2}(\nu)\right\}\right)+O\left(\nu^{\frac{1}{s}\left(1+\frac{1}{s b+1}\right)} \log \nu\right)$

where

$$
c_{b}=\frac{2^{-\frac{(s-1) s b}{2(s b+1)}}}{(\log 2)^{s}}[(b+1) !]^{\frac{-s}{s b+1}} \Gamma\left(1-\frac{1}{s b+1}\right)^{s} .
$$

and $Q(u)$ a periodic function with period 1.

Proof of Theorem 4: We give the proof for $s=2$, and we use one lemma.

\section{Lemma 3:}

Let $V$ be the double series:

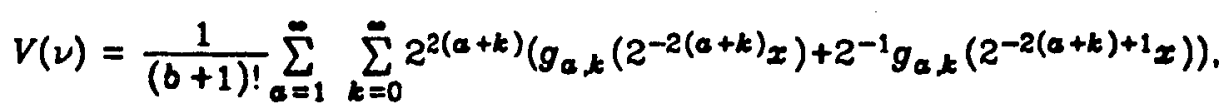

with

$$
g_{a, k}(x)=x^{0+1} f_{b}(x)^{2^{a+1-1}}
$$

Then:

$$
V(\nu)=O\left(\nu^{\frac{1}{2}} \log _{2} \nu\right)
$$

and for any $\nu$ :

$$
|T(\nu)| \leq V(\nu)+N_{H}(\nu)+N_{V}(\nu)-2 .
$$


Theorem 4 follows immediately. We have $T(\nu)=O\left(\nu^{\frac{l}{2}} \log _{2} \nu\right)$ and the order of the leading term of $N_{H}(\nu) \cdot N_{V}(\nu)$ is $\nu^{1+\frac{1}{2 b+1}}$.

\section{Proof of lemma 3 :}

We first prove the inequality. We consider the terms in series $T$ corresponding to $v d>h d$ and note $a=v d-h d, k=k d$. As:

$$
\left\{\begin{array}{c}
f_{0}\left(x 2^{-2 n d-1}\right)^{2^{n d}} \leq 1 \\
f_{0}\left(x 2^{-2 v d}\right)^{2^{w 1}\left(1-2^{-n d}\right)} \leq f_{b}\left(x 2^{-2 v d}\right)^{2 w-1} \\
\left|1-f_{b}(x)^{m}\right| \leq m \frac{x^{b+1}}{(b+1) !}
\end{array}, \text { hd }>0 .\right.
$$

we get the result. When $h d z v d$, we let $a$ be $h d+1-v d$ and $k$ be $v d$.

We derive now the order of magnitude of $V$, which appears to be an harmonic power sum.

Let:

$$
B(\nu)=\sum_{a=1 k}^{\infty} \sum_{=0}^{\infty} 2^{2(\alpha+k)} g_{a, k}\left(2^{-2(\alpha+k)} \nu\right)
$$

Then:

$$
V^{*}(s)=\frac{1+2^{-1-s}}{(b+1) !} B^{*}(s)
$$

Let us compute $B^{\circ}(s)$. Let:

$$
g_{a, k}^{\bullet}(s)=\mathbf{Y}\left(f_{b}(x)^{t} x^{b+1}, s\right) \text { with } t=2^{a+k+1} \text {. }
$$

Using Laplace method to get the asymptotics of $g_{c, k}(s)$, we get:

$$
g_{a, k}(s) \sim \frac{1}{b+1} \Gamma\left(\frac{s+b+1}{b+1}\right) t^{-\frac{s+b+1}{b+1}}+\frac{1}{b+1} \Gamma\left(\frac{s+b+2}{b+1}\right) t^{-\frac{s+b+2}{b+1}} .
$$

We consider the series corresponding to the first terms of this development:

$B_{1}^{*}(s)=\frac{1}{b+1} \Gamma\left(\frac{s+b+1}{b+1}\right) 2^{\frac{s+b+1}{b+1}} \times \sum_{a} 2^{\frac{s(2 b+1)+b+1}{b+1}} \sum_{k}^{k \frac{s(2 b+1)+b+1}{b+1}}$.

This function $B_{1}{ }^{\circ}(s)$ is analytic in the stripe $\left\langle-(b+1),-\frac{1}{2}\left(1+\frac{1}{2 b+1}\right)\right\rangle$ and meromorphic in $\langle-(b+1),+\infty\rangle$. From the second term, we get the series:

$B_{2}(s)=\frac{1}{b+1} \Gamma\left(\frac{s+b+2}{b+1}\right) 2^{\frac{a+b+2}{b+1}} \times \sum_{a} 2^{a \frac{s(2 b+1)+b}{b+1}} \sum_{k}^{k \frac{a(2 b+1)+b}{b+1}}$.

which is analytic in the strip $\left\langle-(b+2),-\frac{1}{2}\left(1-\frac{1}{2 b+1}\right)\right\rangle$. Then $B^{*}$ and $V^{\bullet}$ are analytic in the stripe $\left\langle-(b+1),-\frac{1}{2}\left(1+\frac{1}{2 b+1}\right)\right\rangle$. meromorphic in the stripe $<-(b+2),-\frac{1}{2}\left(1-\frac{1}{2 b+1}\right)>$. Their poles in the first stripe are:

$$
r_{l}=-\frac{1}{2}\left(1+\frac{1}{2 b}\right)+\frac{2 i l \pi}{2 b+1} \frac{(b+1)}{\log 2} .
$$

with the order of multiplicity 2 . Note $p_{l}$ the residues. 


$$
p_{l}=\frac{1}{(b+1) !} \frac{1}{(2 b+1) \log 2} \Gamma\left(1-\frac{1}{2 b+1}+\frac{2 i l \pi}{2 b+1} \frac{1}{\log 2}\right) 2^{1-\frac{1}{2 b+1}}+\frac{2 i l \pi}{2 b+1} \frac{1}{\log 2}
$$

Then:

$$
V(\nu) \sim \nu^{\frac{l}{2}} \log \nu \sum_{l \in \mathbb{Z}} p_{l} e^{\frac{2 i l \pi}{2 b+1}(b+1) \log _{\ell} \nu}=O\left(\nu^{\frac{l}{2}} \log _{2} \nu\right) \text {. }
$$

We may derive the same analysis for biased distributions, for $s=2$.

Theorem 5: The average size of the index of MEH with 2 attributes and biased distribution $(p, q)$ is

$$
S_{U E H}(\nu)=\sum_{k \geq 0} 2^{k}\left(1-\prod_{k_{1}=0}^{k} f_{b}\left(\nu p^{k_{1}} q^{k-k_{1}}\right)\left(\begin{array}{l}
k \\
k_{1}
\end{array}\right)\right.
$$

and satisfies asymptotically:

$$
S_{U E H}(\nu) \sim \frac{\nu^{(b+1) a_{p}}[(b+1) !]^{-a} \Gamma\left(-a_{p}\right) a_{p}}{\log 2} P\left(\left\{(b+1) a_{p} \log _{2} \nu\right\}\right)
$$

where $P(u)$ is a periodic function with mean 1 and $a_{p}=-\frac{1}{\log _{2}\left(p^{b+1}+q^{b+1}\right)}$

Under the same hypotheses, the average size of $M D H$ index has for asymptotic order:

$$
A_{\sigma} \nu^{2(0+1) \sigma}
$$

where $\sigma$ is the smallest real (positive) root of:

$$
\begin{gathered}
\left(p^{b+1}+q^{b+1}\right)^{\sigma}\left(p^{(b+1) \sigma}+q^{(b+1) \sigma}\right)=1 \\
\text { and }\left\{\begin{array}{c}
A_{\sigma}=[(b+1) !]^{-2 \sigma} \Gamma(-\sigma)^{2}\left(p^{b+1}+q^{b+1}\right)^{\sigma}\left(a e^{\sigma a}+b e^{\sigma b}\right)^{-2} \\
a=\log \left(p^{b+1}+q^{b+1}\right)+(b+1) \log p \\
b=\log \left(p^{b+1}+q^{b+1}\right)+(b+1) \log q
\end{array}\right.
\end{gathered}
$$

First, we give some hints for the proof for MDH algorithms. We use the expressions in Theorem 2 . Once more, we replace $\log f_{b}(x)$ by $-\frac{x^{b+1}}{(b+1) !}$. We
get:

$$
E_{V}(\nu) \sim F_{V}(\nu) \text { and } E_{H}(\nu) \sim F_{H}(\nu)
$$

with:

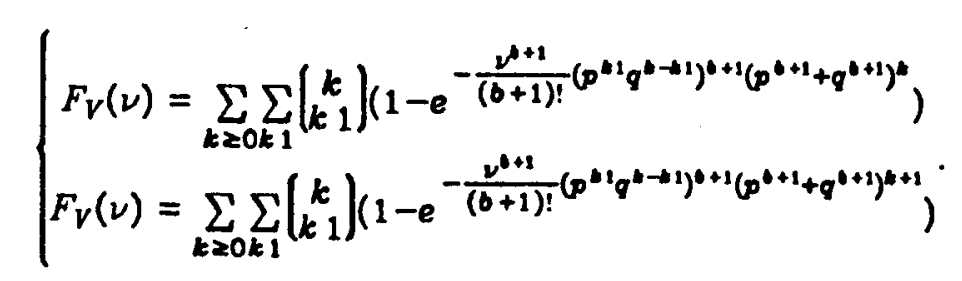

We have: $\mathbf{M}\left(1-e^{-a x^{d+1}}, s\right)=\Gamma\left(\frac{s}{b+1}\right) \cdot a^{-\frac{s}{b+1}}$. Then:

$F^{\cdot}{ }_{v}(s)=-[(b+1) !]^{\frac{s}{b+1}} \cdot \sum_{k}\left(p^{b+1}+q^{b+1}\right)^{-\frac{k s}{b+1}}\left(p^{-s}+q^{-s}\right)^{k}$. 
We set $\sigma=-\frac{s}{b} . F^{*} v^{(s)}$ is analytic when $\Gamma\left(\frac{s}{b}\right)$ and the series are both analytic. i.e. when $0<\sigma<\sigma_{0}$ where $\sigma_{0}$ satisfies the equation above in Theorem 6 .

To compute the expression of the average size of MEH indexes, we consider the probability that no cell of surface $2^{k}$ exist -and there are $\left(\begin{array}{c}k \\ k 1\end{array}\right)$ among them of volume $p^{k 1} q^{k-k 1}$. We get them the desired expression as in the uniform case[3]. The derivation of the asymptotics is quite analogous to the computation in 3.1 .

We are now concerned with robustness. We see first that $a_{p} \rightarrow \frac{1}{b}$ and $\sigma \rightarrow \frac{1}{2 b+1}$ when $(p, q) \rightarrow\left(\frac{1}{2}, \frac{1}{2}\right)$. Thus, $(b+1) a_{p} \rightarrow \frac{b+1}{b}$ and $2(b+1) \sigma \rightarrow 1+\frac{1}{2 b+1}$. The constants involved in Theorem 6: $\frac{[(b+1) !]^{-a} \Gamma\left(-a_{p}\right) a_{p}}{\log 2}$ and $A_{0}$ converge to the corresponding constants in Theorem $A$ and 3 . But in Theorem 3 , the leading term is multiplied by a periodic function $Q$. The periodic terms are associated to a first set of poles of the Mellin transfform regularly distributed on the vertical axis (D). Such a phenomenon seems to disappear for MDH, as Equation (1) has a unique solution with real part $\sigma$.

Nevertheless, a more careful study shows it admits a set of poles "almost regularly distributed on some convex curve (C). (C) is symmetric with respect to the real axis, the intersection with this axis is $\sigma$ and the closest $p$ is to $\frac{1}{2}$, the closest (C) is to (D). Analytically, we have:

$$
S_{U D H}(\nu) \sim \nu^{2(16) \sigma}\left(1+\sum_{l \in Z} p_{l} n^{-\varepsilon_{e}} e^{-2 i l \pi(16) \sigma-\varepsilon] \log l \nu}\right)
$$

which is almost periodical, when $n$ is not too big. Then, MEH and MDH algorithms appear to be robust when the distribution is slightly biased.

Remark: One may derive real valued expressions of the leading terms of asymptotic expansions expressed in Theorem A. Theorem 3 and 4 by their Fourier developments. To get them, we proceed as in [15]. Replacing in the exact expressions $f_{b}(x)$ by the first term of its development in 0 and making a summation when $k$ range in $Z$, we get:

Proposition 5: With the notations of Theorem A. 3 and 4, we have:

$\left\{\begin{array}{c}N_{V}(\nu) \sim \nu^{\frac{\iota}{2}} 2^{-u} \sum_{k \in Z} 2^{-k}\left(1-e^{-u k}\right) \\ N_{H}(\nu) \sim \nu^{\frac{\zeta}{2}} 2^{-u-1} \sum_{k \in Z} 2^{-k}\left(1-e^{-\nu k}\right) \\ S_{\nu D H}(\nu) \sim N_{V}(\nu) \times N_{H}(\nu)\end{array}\right.$

where $u=\left\{\frac{b+1}{2 b+1} \log v\right\}, u k=\frac{2^{u(2 b+1)}}{(b+1) !} 2^{k(2 b+1)}, v k=2^{b+1} \frac{2^{u(2 b+1)}}{(b+1) !} 2^{k(2 b+1) .}$

$S_{U E H}(\nu) \sim \nu^{1+\frac{1}{6}} \cdot 2^{u} \cdot \sum_{k \in Z} 2^{k}\left(1-e^{-w k}\right)$.

where $u=\left\{\left(1+\frac{1}{b}\right) \log \nu\right\}, w k=\frac{2^{b u} 2^{-k b}}{(b+1) !}$. 


\section{Comparison of YIXH and VDH:}

As it was pointed out in Section 1., the occupation of secondary memory by the data is the same for both algorithms. Thus, we compare in this section the sizes of directories. We first assume a uniform distribution. We compare the asymptotic expansions and the transient phases (i.e. the exact values for small $n$ ) for different $b: b=10$ or 50 . Then, we study the robustness of these algorithms when the data distribution is slightly biased.

\subsection{Uniform Distributions:}

The average size of the directory of Extendible Hashing, $S_{b}(\nu)$, has been studied in [5,15] under Bernoulli and Poisson hypotheses for uniform distributions. Asymptotically, we have:

$$
S_{U E H}(\nu)=A_{b} \cdot \nu^{1+\frac{1}{6}}
$$

where $A_{b} \approx \frac{e}{b \log 2}$. In the last section, we have proved a similar result for MDH:

$$
S_{U D H}(\nu) \sim C_{b} \nu^{\frac{\zeta}{2}}
$$

where $c_{b} \approx \frac{e}{b} \cdot \frac{1}{\sqrt{2}(\log 2)^{2}}$. We now compare these asymptotical results and the transient phases.

If we compare the orders of asymptotic expansions of $S_{V E H}$ and $S_{Y D H}$. MDH appears to be asymptotically better than MEH. To conclude, we must consider the constants involved and see how the real expressions converge to these asymptotics. We have computed asymptotics of $S_{\text {UDH }}$ and $S_{\text {UEH }}$ using the MACSYMA system from the expressions in Proposition 5. The corresponding graphs are curves (1) and (2) in Figures 4.a. and 4.b. where $b=10$ and $b=50$. On the first axis, $\nu$ varies from $2^{4}$ to $2^{12}$ (or from $2^{6}$ to $2^{15}$ for $b=50$ ).

We remark that the two graphs coincide when $b$ is 50 . This result is general when $b$ is big, as $\frac{\nu^{1+\frac{1}{b}}}{\nu^{b}}$ remains close to 1 , even for large $\nu$. Moreover. the constants involved are almost equal as: $\frac{A_{b}}{C_{b}} \approx 2^{\frac{1}{2}} \log 2 \approx 1$.

When $b$ is smaller:b $=10$, the curves are quite distinct and MDH seems better. To conclude, we study transient phases in Figures 4.a. and 4.b. for $b=10$ and 50. The curves (3) and (4) correspond to MDH and MEH. We plot the sizes of the directories obtained by 30 simulations as a function of $\nu$ which varies from $2^{4}$ to $2^{12}$ (or $2^{6}$ to $2^{15}$ ). In any case, MDH appears to be better. The difference is about $10 \%$ when $b=50,30 \%$ when $b=10$.

Remark: We have proved that $\frac{S_{\nu D H}(\nu)}{\nu^{\frac{\zeta}{2}}}$ is a periodic function of $\left\{\frac{\zeta}{2} \log (\nu)\right\}$ with period 1. But we see that:

$$
Q\left(\left\{\frac{\zeta}{2} \log \nu\right\}\right) \approx R(\{\zeta \log \nu\})
$$

where $R$ has 1 for period.To derive this, we notice that: 
$Q(u)=A\left(\sum_{l \in Z} c_{l} e^{-2 u \pi u}\right)\left(\sum_{l \in Z} c_{l} e^{-2 u \pi\left(u+\frac{b+1}{2 b+1}\right)}\right)$.

When $\frac{b+1}{2 b+1} \approx \frac{1}{2}, e^{-i \pi \xi \approx(-1)^{l}}$ when $l$ is small. As $c_{l}$ decreases rapidly to 0 :

$$
Q(u) \approx B \sum_{l \in Z} d_{l} e^{-4 i l \pi u}
$$

and has for period $\frac{1}{2}$. This makes the result more precise as $\frac{S_{\mu D H}(\nu)}{\nu^{\frac{l}{2}}}$ appears as a (nearly) periodic function of $\{d o \log (\nu)\}$. Thus, when $b$ is big enough, $\frac{S_{M D H}(\nu)}{\nu^{\frac{1}{2}}}$ and $\frac{S_{\nu E H}(\nu)}{\nu^{\frac{6+1}{2 b}}}$ seem to have the same period (see Figures in Section 4). In particular. $S_{U D H}(\nu)$ and $S_{U E H}(\nu)$ are multiplied by 2 when $\zeta \log \nu$ (resp. $\left.\left(1+\frac{1}{6}\right) \log \nu\right) \approx \log \nu$ increases by 1 .

\subsection{Biased Distributions:}

We assume now that the distribution is slightly biased (see 2.1.) and that $s=2$. We have already considered the robustness of asymptotic expansions. To compare the algorithms in the transient phases, we have computed the expressions of the average sizes of directories for $p \neq q$, given in Theorem 5 for MEH and Theorem 1 for MDH. In Figures 4.c. (resp. 4.d.), we plot the sizes of directories for $\mathrm{MEH}$ when $b=100$ (resp. for $\mathrm{MDH}$, when $b=50$ ). Curves (1). (2), (3) and (4) are associated to $(p, q)=(0.5,0.5),(0.48,0.52),(0.45,0.55)$, $(0.4,0.6)$. It appears that the performances of $\mathrm{MEH}$ quickly deteriorate. For $p=0.45$, the size of the directory is already twice the one we get when $p$ is 0.5 or 0.48 . For $\mathrm{MDH}$, see Figure 4.d., the performances deteriorate more slowly and remain good enough even for $p=0.45$. This may be seen also by studying an other parameter of interest, the ratio $\frac{S}{(n / b)}$ of the directory size to the number of pages necessary for a perfect packing: $\frac{n}{b}$. In Table 1, we give this ratio for different $b$ and $p$ when $n=60000$. The upper (resp. lower) number is MDH (resp. MEH) ratio.

\begin{tabular}{|c|l|l|l|c|}
\hline$p$ & 0.5 & 0.48 & 0.45 & 0.4 \\
\hline \multirow{2}{*}{$b=50$} & 1.705 & 1.937 & 2.749 & 4.459 \\
\cline { 2 - 5 } & 2.382 & 3.348 & 5.125 & 18.38 \\
\hline \multirow{2}{*}{$b=100$} & 1.676 & 1.699 & 2.386 & 3.985 \\
\cline { 2 - 5 } & 1.704 & 2.326 & 3.592 & 14.19 \\
\hline
\end{tabular}

Table 1: Ratio $\frac{58}{n}$ when $n=60000$.

As a conclusion, we may say that MDH should be used when $b$ is small (say $b<20$ ). When $b$ is bigger, $\mathrm{MEH}$, which is easily implemented, is to be chosen when the distribution is uniform. If the distribution is slightly biased $(0.45 \leq p \leq 0.55)$ the performances of MDH are much better. When the data obey 
rather non uniform distributions, one should use a hashing function in order to get a (nearly) uniform distribution.

\section{References}

1. W.A. Burkhard, "Interpolation-Based Index Maintenance," BIT 23 pp. 274-294 (1983).

2. Doetsch, Handbuch der Laplace Pransformation, Birkhauser (1950).

3. R. Fagin, J. Nievergelt, N. Pippenger, and H.R. Strong, "Extendible Hashing:A Fast Access Method for Dynamic Files," ACM TODS 4.3 pp. 315344 (1979).

4. W. Feller, An Introduction to Probability Theory and its Applications. Wiley-third Edition-1971 (1957).

5. Ph. Flajolet, "On the Performance Evaluation of Extendible Hashing and Trie Searching," Acta Informatica 20 pp. 345-369 (1983).

6. Ph. Flajolet, M. Régnier, and R. Sedgewick, "Mellin Transform Techniques for the Analysis of Algorithms ." in preparation. (1985).

7. P. Henrici, Applied and Computational Complex Analysis, Wiley (1977).

8. J.W.Lloyd and K. Ramamohanarao, "Partial-Match Retrieval for Dynamic Files," BIT 22 pp. 150-168 (1982).

9. P.A. Larson, "Dynamic Hashing," BIT 18 pp. 184-201 (1978).

10. H. Mendelson, "Analysis of Extendible Hashing," IEEE Trans, on Software Engineering, pp. 611-624 (1982).

11. T.H. Merrett and E.J. Otoo, "A Storage Scheme for Extendible Arrays," Computing 31 pp. 1-9 (1983).

12. J. Nievergelt, H. Hinterberger, and K.C. Sevcik, "The Grid-file: an Adaptable Symmetric Multi-Key File Structure," ACM TODS 9,1(1984).

13. C. Puech and Ph. Flajolet. "Tree Structure for Partial Match Retrieval," INRIA Research Report 233(1983). submitted to JACM

14. M. Régnier, "On the Average Height of Trees in Digital Search and Dynamic Hashing ." IPL 13 pp. 64-66 (1981).

15. M. Régnier, "Evaluation des performances du hachage dynamique," These de 3-eme cycle, Universite d'Orsay, (1983).

16. M. Tamminen, "The Extendible Cell Method for Closest Point Problems," BIT 22 pp. 27-41 (1982).

17. A.C. Yao, "A Note on the Analysis of Extendible Hashing," IPL 11 pp. 84-86 (1980). 


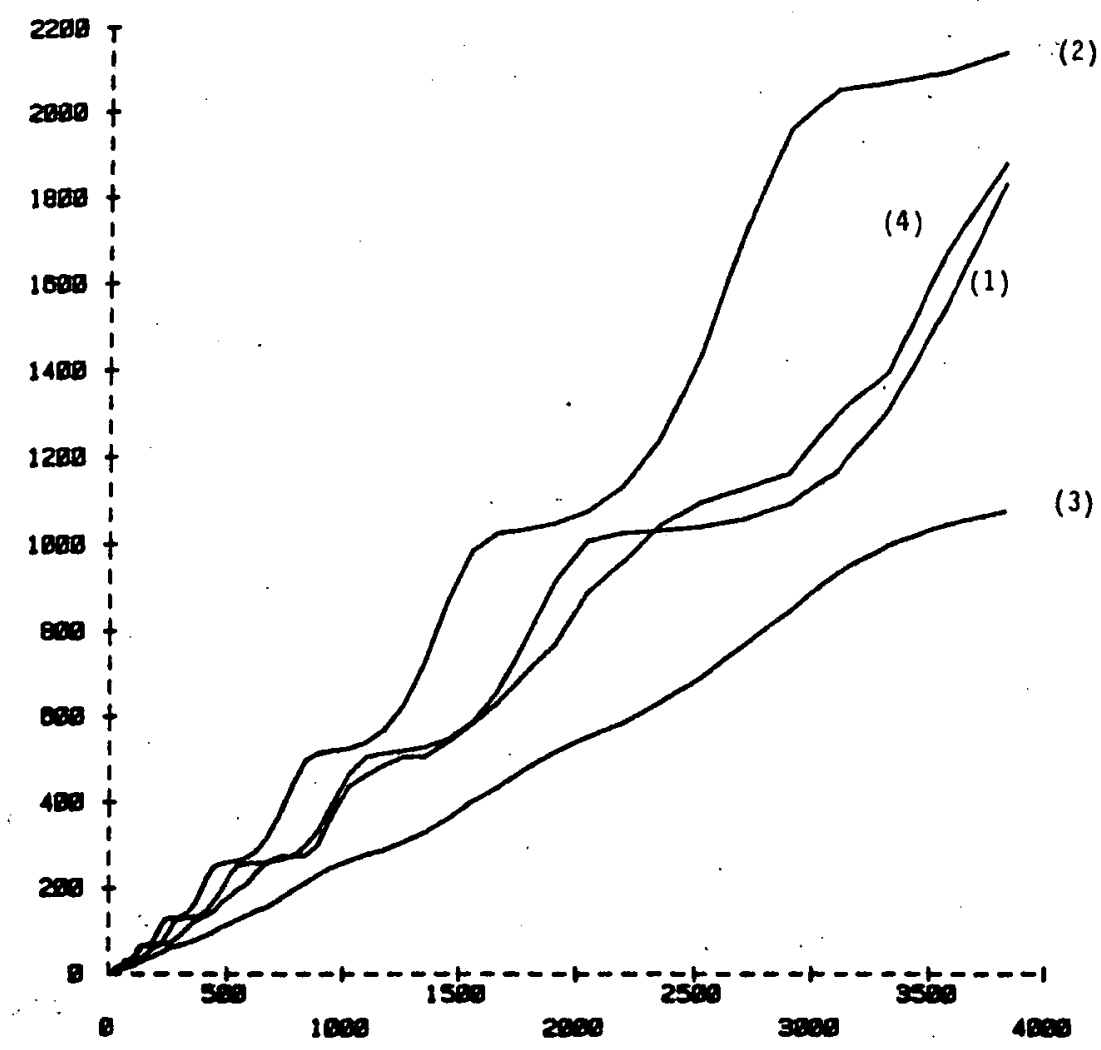

Figure 4.a. : MrH and row directory stzes then be10

(1) and (2) : Assuptotic values for how and KEH

(3) and (4) : Stulations for WOH and $\mathrm{KH}$

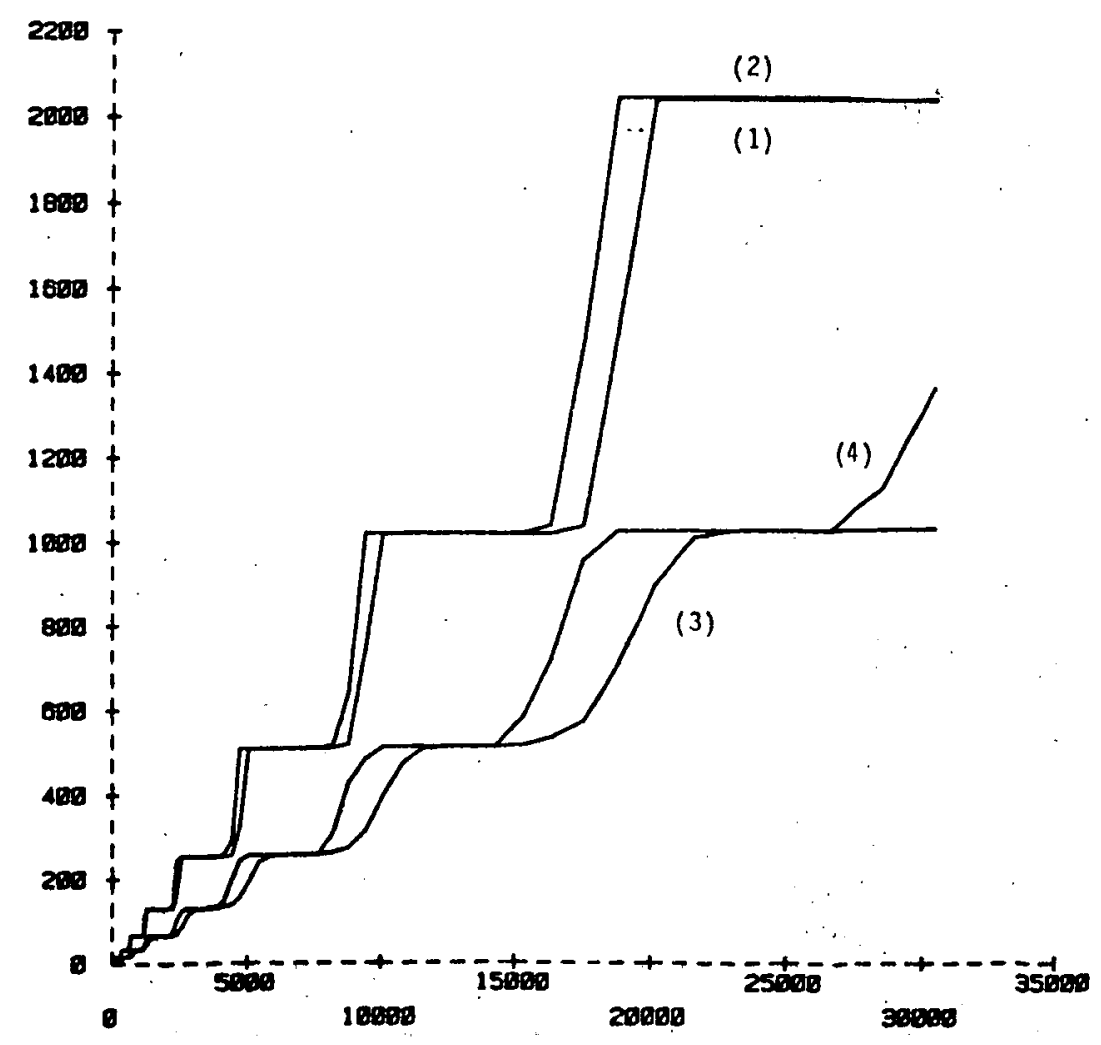

rigore 4.b. : WOH and MCH directory sizes then ba 50 


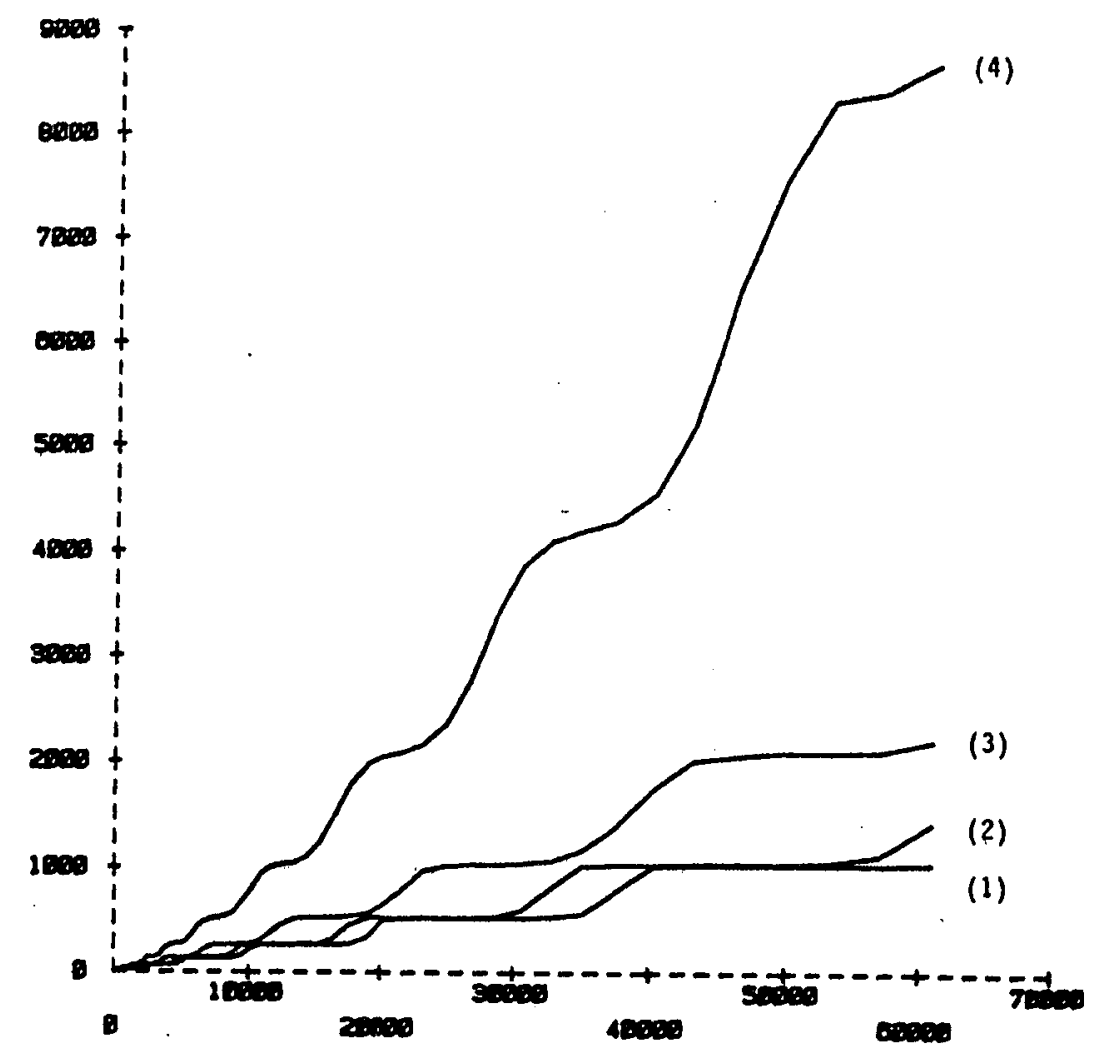

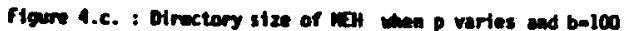

In curves (1). (2). (3) and (4), p=0.5, 0.48, 0.45 and 0.4

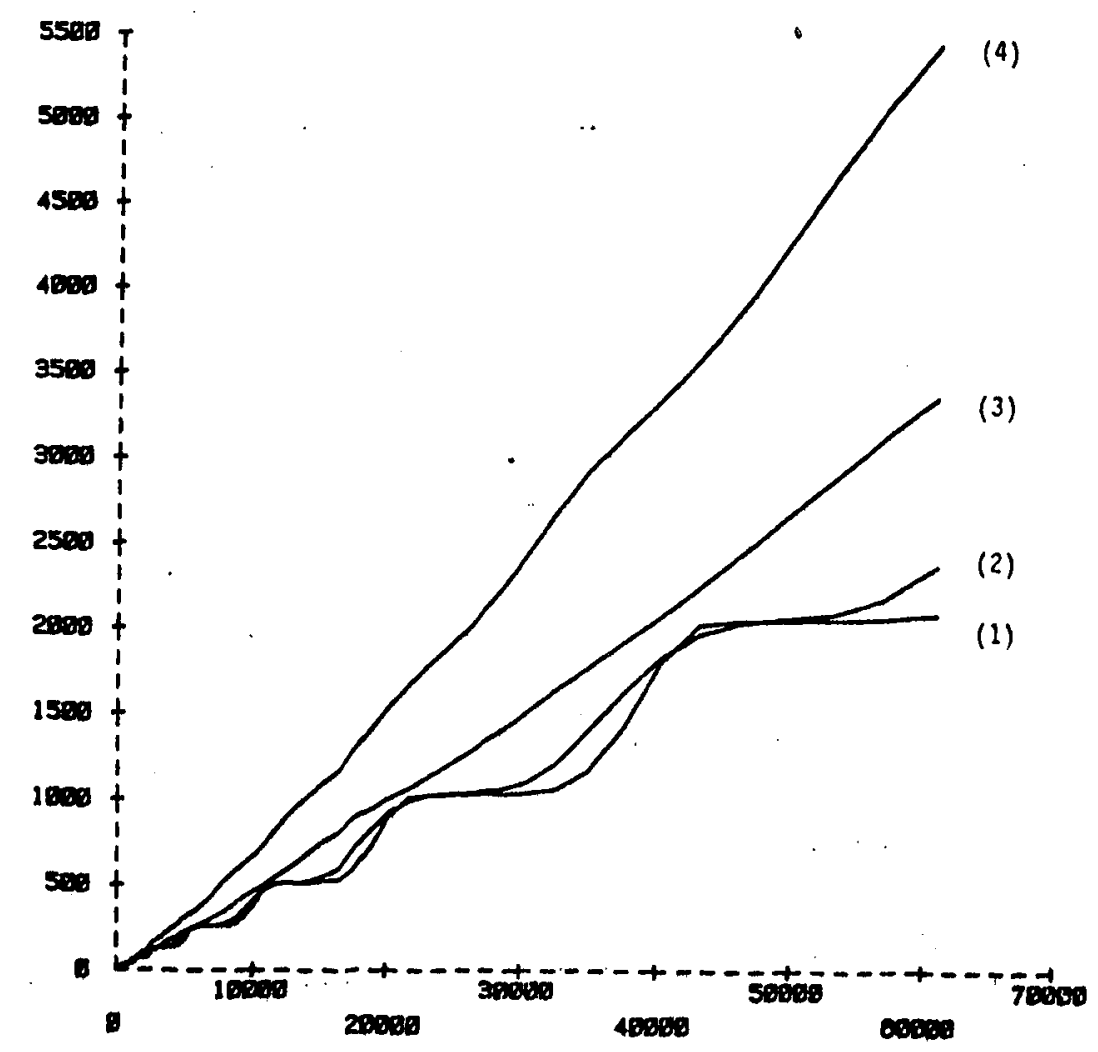

Figure 1.d. : Olmectory size of MDH when D varies and b-50

In curres (1), (2), (3) and (4), 0=0.5, n. $49,0.45$ and $n .4$ 


\section{Imprịmé en France}

I'Institut National de Recherche en Informatique et en Automatique 
